\title{
A Rare Cause of Hemoptysis in West Syndrome- Isolated Aortopulmonary Collaterals in Structurally Normal Heart
}

\author{
Kramadhari Harshith ${ }^{1}$ Ayyappan Anoop ${ }^{1}$ Valakkada Jineesh $^{1}{ }^{10}$ \\ ${ }^{1}$ Department of Imaging Sciences and Interventional Radiology, Sree \\ Chitra Tirunal Institute for Medical Sciences and Technology, \\ Trivandrum, Kerala, India \\ Address for correspondence Anoop Ayyappan, MD, PDCC, \\ Department of Imaging Sciences and Interventional Radiology, Sree \\ Chitra Tirunal Institute for Medical Sciences and Technology, \\ Trivandrum, Kerala 695011, India (e-mail: anoop123a@gmail.com).
}

Indian J Radiol Imaging 2021;31:745-747
Abstract Keywords
- aortopulmonary collaterals
- hemoptysis
- isolated collaterals
- MAPCA coiling
- West syndrome

\begin{abstract}
Major aortopulmonary collateral arteries (MAPCAs) are abnormal systemic to pulmonary collateral vessels originating from the persistent segmental arteries. The common conditions concomitant with MAPCA are congenital heart diseases with reduced pulmonary blood flow. Isolated MAPCAs represent occurrence of collaterals in the absence of underlying heart disease, which commonly present as heart failure, recurrent respiratory tract infection, and pulmonary artery hypertension. Here, we describe a case of West syndrome presenting with hemoptysis due to isolated MAPCAs and its causal relation and management.
\end{abstract}

\section{Introduction}

Major aortopulmonary collateral arteries (MAPCAs) are systemic to pulmonary collateral vessels that arise from the aorta or its first-order branches. MAPCAs usually occur in cases of cyanotic congenital heart diseases with insufficient pulmonary blood flow where they form an important source of accessory blood supply to the lungs. MAPCAs are similar to systemic arteries in histopathology and demonstrate reactivity and stenosis in tortuous locations over time. ${ }^{1}$ These vessels vary in number, origin, and they join either central, lobar, or segmental pulmonary arteries after taking a tortuous course resulting in unpredictable arborization patterns within the lung. The number and size of these collaterals are inversely related to the size of the pulmonary arteries, and they supply the areas of the lung that have a deficient pulmonary blood flow. ${ }^{2}$

Isolated MAPCAs have been described in the literature as presenting with features of left to right shunt. Most of these cases are observed in premature infants presenting as recur- rent respiratory tract infections, congestive cardiac failure, and pulmonary artery hypertension. ${ }^{3-5}$ West syndrome is a seizure disorder in children that is characterized by spastic spells, characteristic electroencephalographic findings, and developmental regression. ${ }^{6}$ Hemoptysis in West syndrome is a very rare clinical presentation, and the reason for such an occurrence is described here.

\section{Case History}

We report a 4-year-old male child, born of normal full-term vaginal delivery to nonconsanguineous parents. There was no history of any drug intake by the mother during the course of her pregnancy. The child had delayed cry after birth and developed jaundice on the 3rd day of life for which phototherapy was given for 5 days and discharged. The patient started having seizures from 1 month of age. On detailed neurological evaluation, he was diagnosed as a case of cryptogenic West syndrome (with all features of triad) and was started on antiepileptic drugs. There was a minimum of
DOI https://doi.org/ 10.1055/s-0041-1735865. ISSN 0971-3026.

\footnotetext{
(C) 2021. Indian Radiological Association. All rights reserved. This is an open access article published by Thieme under the terms of the Creative Commons Attribution-NonDerivative-NonCommercial-License, permitting copying and reproduction so long as the original work is given appropriate credit. Contents may not be used for commercial purposes, or adapted, remixed, transformed or built upon. (https://creativecommons.org/ licenses/by-nc-nd/4.0/)

Thieme Medical and Scientific Publishers Pvt. Ltd., A-12, 2nd Floor, Sector 2, Noida-201301 UP, India
} 
four episodes of seizures per day, each lasting 5 to 10 seconds and was not associated with loss of consciousness. Hemoptysis started from the age of 8 months, with $\sim 20 \mathrm{~mL}$ of blood per episode as multiple episodes. There was no previous history of chronic cough, bluish discoloration, forehead sweating, or recurrent episodes of hospital admissions for respiratory problems. The baby was admitted, and clinical examination was essentially normal. Further evaluations with chest X-ray and echocardiogram were also normal. Laboratory investigations were normal. A computed tomography (CT) scan was done that revealed two major aortopulmonary collaterals ( $4 \mathrm{~mm}$ diameter each) arising from D5 to D6 vertebral levels of descending thoracic aorta supplying the right middle and lower lobe and the left lower lobe, without any congenital cardiac anomaly. There were normalsized pulmonary arteries (diameter of $11 \mathrm{~mm}$ for right and $11 \mathrm{~mm}$ for left pulmonary artery) without evidence of any focal or diffuse stenosis and normal branching in both the lungs (-Figs. 1B and 2B). Magnetic resonance imaging (MRI) brain revealed bilateral periventricular white matter hyperintensities (-Fig. 1A).

The child underwent endovascular coiling of the hypertrophied collaterals arising from the D5 vertebral level of descending thoracic aorta supplying the segments in the left lower lobe and the right middle and lower lobes (-Fig. 1B and $\mathbf{C}$ ). Two large collaterals supplying the right lung and the left lower lobe were embolized by using 0.018 -inch pushable fibered coils (-Fig. 1D). That episode of hemoptysis was
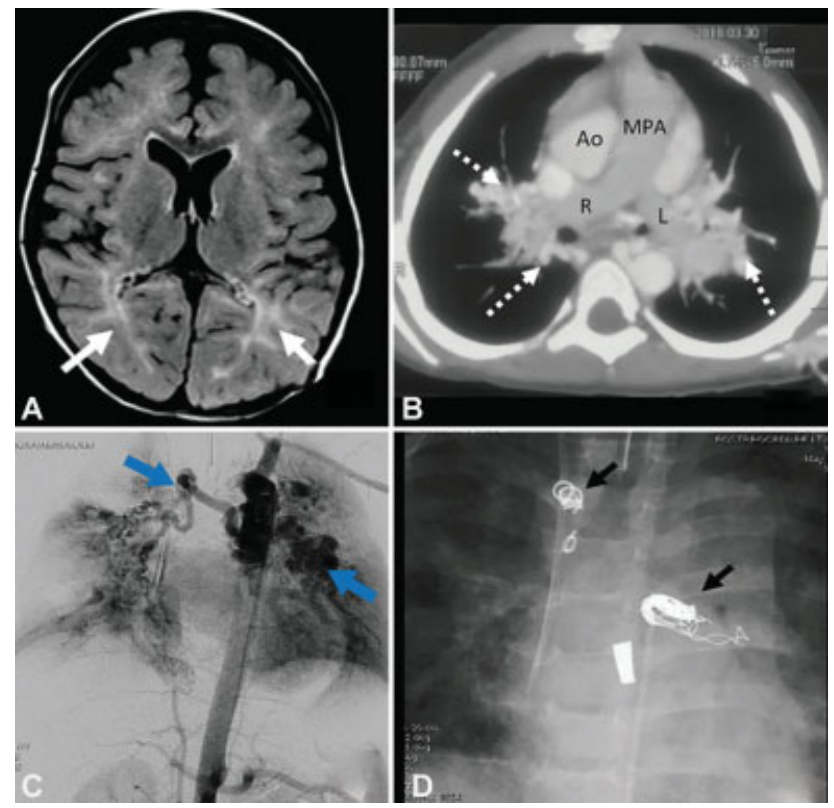

Fig. 1 (A) Axial T2 fluid-attenuated inversion recovery image of the brain shows diffuse bilateral periventricular white matter hyperintensities (arrows). (B) Axial contrast-enhanced computed tomography chest with mildly enlarged central pulmonary arteries without stenosis and predominant major aortopulmonary collateral arteries (MAPCAs) to the right middle and lower lobe and the left lower lobe (dotted arrows). (C) Catheter aortic angiogram shows MAPCAs arising from D5 vertebral level of descending thoracic aorta to the right middle and lower lobes and the left lower lobe (blue arrows) with coil occlusion of these branches as shown in chest X-ray image (D).
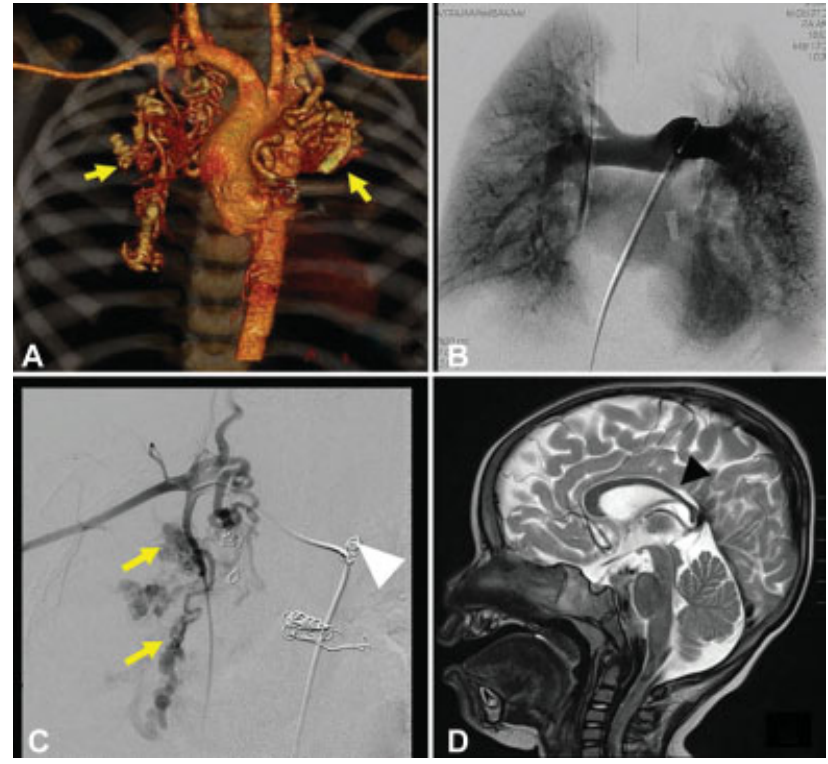

Fig. 2 (A) Volume-rendered computed tomography aortic angiogram after 1 year of initial major aortopulmonary collateral artery (MAPCA) coiling shows multiple collaterals arising from bilateral proximal subclavian artery branches supplying bilateral upper lobes (yellow arrows). Catheter pulmonary angiogram (B) before coiling shows normal diameter of pulmonary branches. (C) Digital subtraction angiography images of right subclavian artery injection showing the new MAPCAs (yellow arrows) prior to coiling and coiled MAPCA of the left subclavian artery branch (white arrowhead). (D) T2 sagittal sections of the brain at 1-year follow-up show the thinned-out corpus callosum (arrowhead).

controlled, and the child was discharged in a stable condition. On follow-up after 1 year, there were repeated episodes of hemoptysis, and hence a repeat contrast CT angiogram was done. CT showed the recruitment of new collaterals measuring 4 and $5 \mathrm{~mm}$ in diameters from ipsilateral subclavian artery branches supplying the bilateral upper lobes, while there was complete obliteration of the previous occluded MAPCAs (-Fig. 2A). Selective subclavian artery angiograms were performed and three large collaterals supplying the right and left upper lobe segments were embolized using a total of eight pushable and detachable 0.018-inch coils (-Fig. 2C). Postprocedure angiograms showed complete obliteration of the blush in the lung parenchyma. The postprocedure period was uneventful with no new episodes of hemoptysis. The child was discharged in a stable condition and on follow-up, there were no new episodes of hemoptysis till 1 year.

\section{Discussion}

Cyanotic congenital heart diseases that have reduced pulmonary blood flow are the common conditions associated with aortopulmonary collateral arteries. These collaterals form the source of pulmonary blood flow in the segment with poor perfusion. These collaterals are the segmental arteries that regress by day 50 of fetal life with the development of the sixth arch and the ductus arteriosus. ${ }^{7}$ They can persist even after birth, especially in premature babies, which regress without any obvious 
treatment. ${ }^{8}$ Isolated collaterals that persist without underlying heart disease are rare but have been described in the literature. Prematurity, neonatal hypoxic and ischemic episodes, traumatic conditions, and ingestion of maternal antiepileptic drugs have been described to have an association with this condition. ${ }^{9,10}$ These patients present with pulmonary artery hypertension, recurrent respiratory tract infection, bronchopulmonary dysplasia, and congestive cardiac failure due to the left to right shunt. ${ }^{3-5}$ Early identification and closure of these collaterals with either surgical or endovascular means are warranted to prevent microvascular changes in the lung parenchyma. The abnormal vessels generally arise from the thoracic aorta but can arise from the subclavian arteries, abdominal aorta, or the celiac artery. ${ }^{7}$ Our case is unique as isolated MAPCAs causing hemoptysis as primary presentation have not been described previously in the literature. Significant parenchymal supply and multiplicity ruled out hypertrophied bronchial arteries. The coexistence of West syndrome and isolated MAPCAs is not described. West syndrome being a cause for multiple seizure episodes and the occurrence of hypoxic episodes associated with seizures is the only possible explanation for MAPCAs, which was substantiated in the follow-up MRI as thinning of corpus callosum (-Fig. 2D). There were no prematurity or recurrent chest infections or maternal intake of any antiepileptic drugs in the index case. Acherman et al have reported $66 \%$ of premature infants have MAPCAs. ${ }^{7}$

These collaterals can be surgically ligated or can be occluded by the endovascular route, which is currently the preferred modality of treatment. Coils and plug devices are used for the treatment of such patients. ${ }^{3}$ The choice of the device depends on the size of the collateral, which can be determined by preprocedural CT/MR angiography. Hemoptysis is an indication for occlusion of the isolated MAPCAs.

Patients with pulmonary artery hypertension require further treatment with pulmonary vasodilators following MAPCA coiling. ${ }^{3}$ In cases presenting with heart failure, control of the symptoms of failure followed by occlusion of MAPCAs is described. ${ }^{5}$

\section{Conclusion}

Isolated MAPCAs can have hemoptysis as one of the presentations. Hypoxic episodes in neonatal periods may trigger the development of isolated MAPCAs. Nonresolving MAPCAs have to be embolized for control of cardiac symptoms.

\section{Source(s) of Support in the Form of Grants}

None.
If the manuscript was presented as part at a meeting, the organization, place, and exact date on which it was read No.

\section{Declaration of Patient Consent}

The authors certify patient's guardian has given his/her consent for the patient's images and other clinical information to be reported in the journal. Review board for publishing the same was also obtained.

\section{Financial Support and Sponsorship \\ Nil.}

\section{Conflicts of Interest}

There are no conflicts of interest.

\section{Acknowledgments}

None.

\section{References}

1 Presnell LB, Blankenship A, Cheatham SL, Owens GE, Staveski SL. An overview of pulmonary atresia and major aortopulmonary collateral arteries. World J Pediatr Congenit Heart Surg 2015;6(04):630-639

2 Latus H, Gummel K, Diederichs T, et al. Aortopulmonary collateral flow is related to pulmonary artery size and affects ventricular dimensions in patients after the Fontan procedure. PLoS One 2013;8(11):e81684

3 Kunwar BK, Paddalwar S, Ghogare M. Large isolated major aortopulmonary collateral artery causing severe pulmonary hypertension in an infant: a rare and challenging diagnosis. J Clin Diagn Res 2017;11(06):OD18-OD20

4 Patra S, Srinivas SK, Agrawal N, Jayaranganath M. Isolated major aortopulmonary collateral artery in an infant presenting with recurrent lower respiratory tract infection. BMJ Case Rep 2013; 2013:bcr2013200421-bcr2013200421

5 Tinmaswala MA, Saple PP, Gupta A, Prachi N, Nitinkumar A, Amin $\mathrm{K}$. Isolated major aortopulmonary collateral artery causing CCF in a newborn: a case report. Int J Med Res Health Sci 2015; 4:471-473

6 D'Alonzo R, Rigante D, Mencaroni E, Esposito S. West syndrome: a review and guide for paediatricians. Clin Drug Investig 2018;38 (02):113-124

7 Acherman RJ, Siassi B, Pratti-Madrid G, et al. Systemic to pulmonary collaterals in very low birth weight infants: color doppler detection of systemic to pulmonary connections during neonatal and early infancy period. Pediatrics 2000;105(3 Pt 1): 528-532

8 Boyden EA. The time lag in the development of bronchial arteries. Anat Rec 1970;166(04):611-614

9 Botenga AS. The significance of broncho-pulmonary anastomoses in pulmonary anomalies: a selective angiographic study. Radiol Clin Biol 1969;38(05):309-328

10 Pelizzo G, Calcaterra V, Mannarino S, Moramarco LP, Leati G, Quaretti P. Aortopulmonary collateral artery in prenatal exposure to carbamazepine - endovascular therapy and technical considerations: a case report. J Med Case Reports 2015;9:183 